\title{
Niemann-Pick(ing) on Ebola
}

\section{By Tracey Baas, Senior Editor}

Teams in Europe and the U.S. have identified a host protein called Niemann-Pick disease type $\mathrm{C} 1$ that is required for Ebola virus to enter cells ${ }^{1,2}$ and both groups are developing small molecules that target the entry protein. Both teams also are pursuing challenge studies in animals with the U.S. Army Medical Research Institute of Infectious Diseases.

Ebola is a filovirus that causes a rapid hemorrhagic fever. In many outbreaks mortality exceeds $75 \%$. The infection is a public health threat in regions of central Africa, where the virus is endemic, but also worldwide due to imported infections and the potential for bioterrorism. There are no effective vaccines or antivirals. The current standard of treatment is supportive care while the body tries to fight the infection.

The two teams went at the problem of

"There seems to be a
wealth of possibilities here
given that three distinct
inhibitors of Ebola have
been reported."
-Duncan Wardrop,
University of Illinois at Chicago

"There seems to be a inhibitors of Ebola have been reported." University of Illinois at Chicago virus entry called cathepsin B (CTSB). ${ }^{3}$

Brummelkamp is a group leader at The Netherlands Cancer Institute and an adjunct principal investigator at the Research Center for Molecular Medicine of the Austrian Academy of Sciences. The work described here was performed while Brummelkamp was a fellow at the Whitehead Institute for Biomedical Research. Chandran is an assistant professor in microbiology and immunology at the Albert Einstein College of Medicine of Yeshiva University; Whelan is an associate professor of microbiology and molecular genetics at Harvard Medical School; Dye is a principal investigator at the U.S. Army Medical Research Institute of Infectious Diseases (USAMRIID).

Cultured cells with mutant NPC1 had less Ebola or Marburg virus infection than wild-type cells. Marburg is a filovirus in the same family as Ebola.

Cells treated with either imipramine or U18666A, two compounds known to cause a cellular phenotype similar to NPC1 deficiency, 4 also had lower infection rates than untreated cells. U18666A is a small molecule cholesterol synthesis inhibitor, and imipramine is a generic antidepressant.

In mouse models of Ebola or Marburg virus infection, heterozygous $N p c 1$ knockout animals had significantly greater survival than wild-type mice ( $p=0.006$ and $p=0.003$, respectively).

Meanwhile, a team led by James Cunningham screened a small molecule library and identified stopping Ebola infection from opposite directions-one looked for host factors the virus interacts with and the other started with a small molecule screen and worked backward to find the targets.

Both identified Niemann-Pick disease type C1 (NPC1) as a requisite for cellular entry (see Figure 1, "Proposed model for Ebola virus entry"). The findings were surprising because the endosomal and lysosomal cholesterol transporter had previously only been associated with a type of lysosomal disorder called Niemann-Pick disease, type C.

In a collaborative effort, the groups of Thijn Brummelkamp, Kartik Chandran, Sean Whelan and John Dye used a genetic screen in a human cell line to identify host factors required for Ebola virus entry-NPC1 was the strongest hit. Two other hits were the homotypic fusion and vacuole protein-sorting (HOPS) complex and a previously known host factor for

Figure 1. Proposed model for Ebola virus entry. The Ebola virus is first taken up and trafficked by endosomes [a]. During this process, the glycoproteins that cover the surface of the virus are cleaved by cathepsin B (CTSB) protease, which removes the heavily glycosylated domains to expose the putative receptor-binding domain of the glycoprotein [b]. Following the fusion of the endosome with the vacuole [c], which contains the Niemann-Pick disease type C1 (NPC1) protein, the virus binds to NPC1 and is released into the cellular cytoplasm [d]. Virus entry can be curbed with NPC1 inhibitors ${ }^{1,2}$ or CTSB inhibitors. ${ }^{3}$ a new benzylpiperazine adamantane diamide-derived compound that decreased several varieties of Ebola infection by about $99 \%$ in vitro compared with vehicle. The researchers then used a cross-linking experiment and determined the compound bound NPC1.

Cunningham is an associate professor of medicine, microbiology and molecular genetics at Brigham and Women's Hospital and Harvard Medical School. He previously identified CTSB's role as a host protein

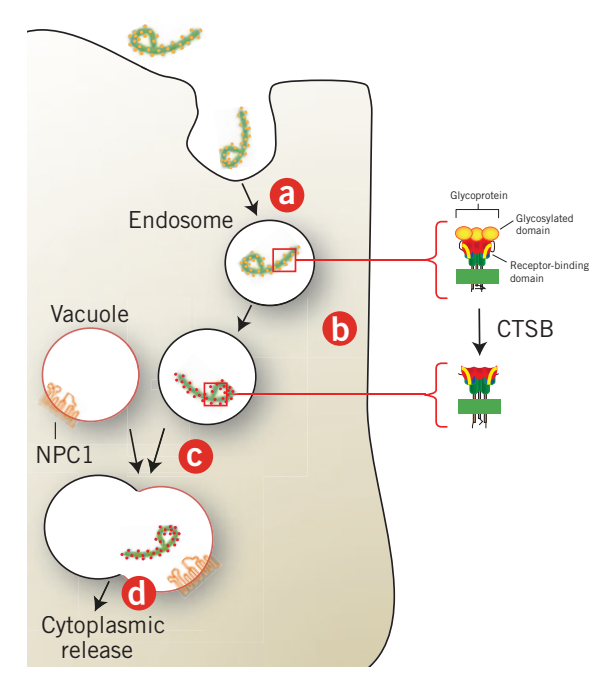




\section{ANALYSIS}

\section{TARGETS \& MECHANISMS}

necessary for Ebola binding.

Both studies were published in Nature.

\section{Going viral}

Duncan Wardrop, associate professor of chemistry at the University of Illinois at Chicago, noted that the identification of cellular receptors for Ebola virus had been slow going. Thus, he thinks the new findings are important, "particularly since the authors find that all filoviral strains, including Marburg, are strictly NPC1 dependent. Moreover, there seems to be a wealth of possibilities here given that three distinct inhibitors of Ebola have been reported," namely the piperazine derivatives, imipramine and U18666A.

For any class of compounds, "it will be important to show that these drugs are able to completely protect mice or guinea pigs against Ebola," said Thomas Geisbert, professor of microbiology and immunology at The University of Texas Medical Branch. "There are quite a few treatments that have been shown to protect rodents against Ebola but fail when tested in the more robust nonhuman primate models."

Geisbert previously collaborated with the USAMRIID when he was at the Boston University School of Medicine to show that small interfering RNA against Ebola could be delivered in stable nucleic acid lipid particles (SNALPs) to protect macaques from Ebola challenge. ${ }^{6}$ The siRNA-SNALP formulations are from Tekmira Pharmaceuticals Corp.

Wardrop's laboratory identified an isoxazole-based compound and synthesized and characterized derivatives that inhibited cell entry of Ebola and Marburg virus. They are in the process of determining the host receptor for their family of inhibitors.

Independently, both Cunningham's lab and Dye and his collaborators will further pursue the use of the compounds in challenge studies with animal models of Ebola infection at USAMRIID.

Cunningham's lab also hopes to determine how NPC1 and the glycoproteins of Ebola interact structurally.

For Cunningham's work, a patent application has been filed covering compositions of compounds and their use to treat Ebola and related infections. The IP is available for licensing through the Harvard University Office of Technology Development.

For the work by Brummelkamp's, Whelan's, Chandran's and Dye's groups, a patent has been filed by the Albert Einstein College of Medicine, Harvard Medical School and the Whitehead Institute covering the host factor targets NCP1 and HOPS, and for the two therapeutic leads imipramine and U18666A as treatments for Ebola infection. The work is available for licensing through the Albert Einstein College of Medicine.

Another patent is held by the Whitehead Institute that covers the genetic screening technology platform. That platform is being developed by Haplogen $\mathbf{G m b H}$, a privately held biotechnology company cofounded by Brummelkamp. Haplogen is using the technology to identify new pathogen host factors to target in infectious diseases.

Baas, T. SciBX 4(35); doi:10.1038/scibx.2011.981

Published online Sept. 8, 2011

\section{REFERENCES}

1. Carette, J.E. et al. Nature; published online Aug. 24, 2011; doi:10.1038/nature10348

Contact: Thijn R. Brummelkamp, Whitehead Institute for Biomedical Research, Cambridge, Mass. e-mail: t.brummelkamp@nki.nl

2. Côté, M. et al. Nature; published online Aug. 24, 2011; doi:10.1038/nature10380

Contact: James Cunningham, Brigham and Women's Hospital, Boston, Mass.

e-mail: jcunningham@rics.bwh.harvard.edu

3. Chandran, K. et al. Science 308, 1643-1645 (2005)

4. Cenedella, R.J. Lipids 44, 477-487 (2009)

5. Rodriguez-Lafrasse, C. et al. Biochim. Biophys. Acta. 1043, 123-128 (1990)

6. Geisbert, T.W. et al. Lancet 375, 1896-1905 (2010)

COMPANIES AND INSTITUTIONS MENTIONED

Albert Einstein College of Medicine of Yeshiva University, New York, N.Y.

Boston University School of Medicine, Boston, Mass.

Brigham and Women's Hospital, Boston, Mass.

Haplogen GmbH, Vienna, Austria

Harvard Medical School, Boston, Mass.

Harvard University, Cambridge, Mass.

The Netherlands Cancer Institute, Amsterdam, the Netherlands Research Center for Molecular Medicine of the Austrian Academy of Sciences, Vienna, Austria

Tekmira Pharmaceuticals Corp. (TSX:TKM; NASDAQ:TKMR), Burnaby, British Columbia, Canada

U.S. Army Medical Research Institute of Infectious Diseases, Fort Detrick, Md.

University of Illinois at Chicago, Chicago, III.

The University of Texas Medical Branch, Galveston, Texas

Whitehead Institute for Biomedical Research,

Cambridge, Mass. 